\title{
Quality of Life, Sexual Functioning, and Physical Functioning Following Perineal Reconstruction with the Lotus Petal Flap
}

\author{
Joke Hellinga, MD ${ }^{1}$, Martin W. Stenekes, MD, PhD $^{1}$, Paul M. N. Werker, MD, PhD ${ }^{1}$, Moniek Janse, PhD $^{2}$, \\ Joke Fleer, $\mathbf{P h D}^{2}$, and Boudewijn van Etten, $\mathbf{M D}, \mathbf{P h D}^{3}$ \\ ${ }^{1}$ Department of Plastic Surgery, University Medical Center Groningen, University of Groningen, Groningen, The \\ Netherlands; ${ }^{2}$ Department of Health Sciences, Section Health Psychology, University Medical Center Groningen, \\ University of Groningen, Groningen, The Netherlands; ${ }^{3}$ Department of Surgical Oncology, University Medical Center \\ Groningen, University of Groningen, Groningen, The Netherlands
}

\begin{abstract}
Background. Lotus petal flaps (LPF) may be used for the reconstruction of extralevator abdominoperineal defects that cannot be closed primarily. Limited data are available on how perineal reconstruction with the LPF impacts on patients' quality of life (QoL), sexual functioning, and physical functioning.

Methods. A cross-sectional study was performed following perineal reconstruction with the LPF. The QoL of patients having undergone LPF reconstruction was compared with a control group in which perineal defects were closed without flaps. Sexual and physical functioning (presence of perineal herniation and range of motion [ROM] of the hip joints) could only be evaluated in the LPF group. Psychometrically sound questionnaires were used. Physical functioning was evaluated subjectively with binary questions and objectively by physical examination. Results. Of the 23 patients asked to participate, 15 (65\%) completed the questionnaires and $11(47 \%)$ underwent physical examination. In the control group, 16 patients were included. There were no significant differences in QoL between the LPF and control groups. Within the LPF group, $33 \%$ of patients were sexually active postoperatively compared with $87 \%$ preoperatively. No perineal herniation was found. The ROM of the hip joints was
\end{abstract}

(C) The Author(s) 2020

First Received: 18 April 2020;

Published Online: 2 July 2020

M. W. Stenekes, MD, PhD

e-mail: m.w.stenekes@umcg.nl bilaterally smaller compared with the generally accepted values.

Conclusions. Conclusions should be made with care given the small sample size. Despite a supposedly larger resection area in the LPF group, QoL was comparable in both groups. Nonetheless, reconstruction seemed to affect sexual function and physical function, not hampering overall satisfaction.

Resection of advanced anal and rectal tumors by means of extralevator abdominoperineal excision (ELAPE) may lead to extensive soft tissue defects in the perineal area. ${ }^{1}$ Wound healing may be impaired due to the application of neoadjuvant radiotherapy and chemotherapy, especially in patients with advanced cancer and malnutrition. ${ }^{1,2}$ When resulting defects are too large for tension-free primary closure, reconstruction with a soft tissue flap should be performed. ${ }^{2,3}$ Such a reconstruction has the potential for bringing well-vascularized, non-irradiated soft tissue into the defects and is able to fill the dead space and minimizes the risk of fluid collections and infections. ${ }^{1,2,4,5}$ Our first choice of flaps following ELAPE is the lotus petal flap $(\mathrm{LPF})^{6}$, a regional flap based on perforators of the internal and external pudendal vessels behind. ${ }^{2-7}$ This flap has been widely used for vulvar reconstruction over the past decades, yet the application for perineal reconstruction is relatively new as its use was reported for the first time in 2007. ${ }^{6,8-10}$

From research on vulvar reconstruction, we know that reconstructive surgery in this area may have a large impact on quality of life (QoL) and sexual functioning. ${ }^{11}$ However, little or no data are available on how perineal 
reconstruction with the LPF impacts on patients' lives (i.e. QoL, sexual functioning, and physical functioning). Perineal herniation has been reported as a common complication (incidence rates vary from 1 to 26\%) following ELAPE. ${ }^{12}$ Reconstruction with a soft tissue flap supposedly lowers the risk of perineal herniation, but currently there are no data available to support this statement. ${ }^{13}$ There are also no data available on the ROM of the ipsilateral hip joint after LPF, whereas it is likely that this procedure influences the function of the hip joint as the LPF donor site is located on the junction of the buttock and the upper thigh and is sometimes quite tight after primary closure.

Therefore, the primary aim of this study was to assess the postoperative QoL of patients who had undergone LPF reconstruction following ELAPE and compare this with a control group in whom primary closure of the perineal defect following abdominoperineal excision (APE) had been possible. The secondary aim was to assess postoperative sexual and physical functioning in both groups. Two aspects of physical functioning had our main interest; first, the presence of perineal herniation, and second, the range of motion (ROM) of the hip joint on the side where the LPF was harvested. Our hypothesis is that QoL in the reconstructive group is higher compared with the control group. Furthermore, we believe that sexual function will be influenced by the reconstruction, but physical functioning will barely be affected.

\section{METHODS}

\section{Patients and Procedures}

The LPF group consisted of patients who underwent perineal reconstruction with the LPF at the University Medical Center Groningen, The Netherlands, between October 2011 and December 2015. Patients who were deceased at the time of follow-up and patients who were not able to read or write Dutch were excluded. Eligible patients received a package containing an information letter, an informed consent form, the questionnaire, and a return envelope. Patients who did not reply within 2 weeks were called by the first author, asking them if they had received the package and asking them to contemplate to return the informed consent and, if applicable, the questionnaire. After QoL data collection was completed, participating patients were asked to visit the outpatient clinic to be physically examined. They were asked to sign informed consent during the visit to the clinic.

The historical control group consisted of colorectal cancer patients who had undergone APE and radiotherapy between December 2011 and March 2013 and did not need reconstruction because of the limited size of the resulting defect after tumor resection allowing for primary closure. These patients were selected from a larger study performed by Janse et al. ${ }^{14}$ investigating QoL in the first 18 months after colorectal cancer.

\section{Measures}

Data regarding patient demographics and treatment and disease characteristics were collected from the medical files.

QoL was assessed in both the LPF and the control group using two psychometrically sound questionnaires. The global health status scale and the five functional scales (i.e. physical, role, emotional, cognitive, and social functioning) of the European Organization for Research and Treatment of Cancer Quality of Life Questionnaire C30 (EORTC QLQ-C30) version 3.0 were used to assess general QoL. ${ }^{15}$ After transformation, the scores ranged from 0 to 100 . In addition, the European Organization for Research and Treatment of Cancer Quality of Life Questionnaire CR29 (EORTC QLQ-CR29), which was specifically developed to assess the QoL of colorectal cancer patients, was used. ${ }^{16,17}$ This questionnaire consists of three functional scales (i.e. body image, anxiety, and weight). After transformation the scores also ranged from 0 to 100 .

Sexual functioning and physical functioning were only assessed in the LPF group. The Female Sexual Function Index (FSFI) and the Male Sexual Function Index (MSFI) were used to assess sexual functioning of the female and male patients, respectively. ${ }^{18-20}$ Only the total score was used (maximum of 36 points). A total score $>26$ is considered as normal sexual functioning. ${ }^{21}$ In addition, binary questions were used to assess whether or not patients (1) currently had a partner; (2) were sexually active before surgery; (3) were sexually active after surgery; and (4) had intercourse after surgery. Sexual activity was permitted 6 weeks postoperatively. Postoperative sexual activity was also assessed in the control group.

LPF-specific physical functioning was measured subjectively and objectively. Subjective physical functioning was measured by asking whether or not patients experienced (1) any perineal bulging, reflecting perineal herniation (during sitting, standing or walking); (2) limitations in the use of the left hip (during sitting or walking); (3) limitations in the use of the right hip (during sitting or walking); (4) other limitations during sitting; and (5) limitations during cycling. Objective physical functioning was measured by determination of the active ROM (flexion) in both hips (using a goniometer) according to the standard way of measuring of the American Academy of Orthopaedic Surgeons. ${ }^{22}$ Of patients with bilateral donor sites, the ROM of both sides was averaged. 
The existence of perineal herniation was examined by the first author in both the lateral and standing positions. In the standing position, the Valsalva maneuver was performed. Postoperative perineal herniation was defined as the protrusion of intra-abdominal viscera through a defect in the pelvic floor into the perineal region. In the case of perineal herniation, the extent was estimated as the distance to the normal level of the buttocks.

\section{Data Analysis}

The IBM Statistical Package for the Social Sciences (SPSS) version 23 was used for the analyses. Data for the LPF group were compared with the control group using the Mann-Whitney U-test or Chi square test. The effect of the reconstruction on the EORTC QLQ-C30 and CR29 data was determined using linear regression. Treatment (reconstruction or no reconstruction) and all significant differences in study characteristics (tumor classification, excision type, type of [chemo]radiotherapy, and time between surgery and receiving back the survey) (Table 1) were included as fixed variables, and the EORTC scale was included as a random variable. A $p$ value $<0.05$ was considered statistically significant.

Descriptive data on physical and sexual functioning were reported as $n(\%)$ or median (range). In the FSFI/ MFSI questionnaires, the domain scores were considered missing in case more than half of the items of that domain were missing, otherwise missing items were replaced by the mean of the known items.

\section{RESULTS}

\section{Descriptives}

Thirty-five patients underwent reconstruction of a perineal defect with LPF between 2011 and 2015. Eleven patients were deceased at the time of study and one patient

TABLE 1 Study characteristics

\begin{tabular}{|c|c|c|c|}
\hline & Lotus petal flap group [ $n=15]$ & Control group $[n=16]$ & $p$-Value \\
\hline Age, years [mean $(\mathrm{SD})]$ & $60.4(13.6)$ & $63.7(9.9)$ & 0.64 \\
\hline Sex, male & $12(80.0)$ & $11(68.8)$ & 0.47 \\
\hline Tumor type & & & 0.17 \\
\hline Rectal cancer & $12(80.0)$ & $16(100)$ & \\
\hline Anal cancer & $2(13.3)$ & $0(0)$ & \\
\hline Giant condylomata & $1(6.7)$ & $0(0)$ & \\
\hline Tumor classification & & & 0.04 \\
\hline $\mathrm{T} 1$ & $2(13.3)$ & $0(0)$ & \\
\hline $\mathrm{T} 2$ & $3(20.0)$ & $8(50.0)$ & \\
\hline $\mathrm{T} 3$ & $6(40.0)$ & $8(50.0)$ & \\
\hline $\mathrm{T} 4$ & $4(26.7)$ & $0(0)$ & \\
\hline Excision type & & & 0.001 \\
\hline APE & $0(0)$ & $16(100)$ & \\
\hline ELAPE & $4(26.7)$ & $0(0)$ & \\
\hline ELAPE + excision sacrum & $4(26.7)$ & $0(0)$ & \\
\hline Total exenteration & $4(26.7)$ & $0(0)$ & \\
\hline Total exenteration + excision sacrum & $2(13.3)$ & $0(0)$ & \\
\hline Total colectomy + rectal amputation & $1(6.7)$ & $0(0)$ & \\
\hline Radiotherapy & $15(100)$ & $16(100)$ & NA \\
\hline Type of (chemo)radiotherapy & & & 0.01 \\
\hline Long-course chemoradiation & $13(86.7)$ & $7(43.8)$ & \\
\hline Short-course radiotherapy $(5 \times 5$ Gy $)$ & $1(6.7)$ & $9(56.3)$ & \\
\hline Previous radiotherapy & $1(6.7)$ & $0(0)$ & \\
\hline Time between surgery and survey, months [median (range)] & $30.6(16.4-64.3)$ & $16.1(13.5-21.2)$ & $<0.001$ \\
\hline
\end{tabular}

$A P E$ abdominoperineal excision, ELAPE extralevator abdominoperineal excision, $S D$ standard deviation, $N A$ statistical analyses could not be performed due to a $100 \%$ score in both groups

Data are expressed as $n(\%)$ unless otherwise specified 
was excluded because they were not able to read or write Dutch. In total, 23 patients were asked to participate. Fifteen patients $(65.2 \%)$ returned the questionnaire and 11 of the 23 patients $(47.8 \%)$ agreed to participate in the physical examination. Figure 1 shows the pre-, inter- and postoperative result of reconstruction with the LPF in a typical case.

Four patients $(26.7 \%)$ did not have any postoperative complications; seven patients $(46.7 \%)$ had a wound dehiscence that healed without further surgical intervention; and four patients $(26.7 \%)$ had a complication where surgical intervention was required: two patients (13.3\%) had partial necrosis of the flap and underwent necrotomy and additional reconstruction with another LPF. The other two patients (13.3\%) had an abscess and underwent surgical incision and drainage. No late complications due to radiotherapy were reported.

\section{Quality of Life}

The LPF and control groups did not significantly differ in mean age and sex, but patients in the LPF group had significantly larger tumors and had undergone more extensive treatment (Table 1). One female patient also underwent a posterior vaginal wall reconstruction with the LPF. In the control group, no biological mesh was used, while in the LPF group, both an omentoplasty and biological mesh were used as part of reconstruction of the pelvic floor in 10 patients $(66.7 \%)$. In three patients (20.0\%), an omentoplasty only was used, and, in one patient $(6.7 \%)$, a biological mesh only was used, leaving one patient $(6.7 \%)$ with neither an omentoplasty nor a biological mesh. No biological mesh-related complications were reported. The LPF group showed a significantly longer time between surgery and the return of the completed survey $(p<0.001)$.
There were no significant differences between both groups in terms of general QoL scores (EORTC QLQ-C30) and colorectal-specific QoL scores (EORTC QLQ-CR29) [Table 2]. The global health status and some of the functional scales of the EORTC QLQ-C30 (physical functioning, emotional functioning, and cognitive functioning) showed a positive reconstruction effect. Although those differences were not significant in our small study population, it shows a trend towards higher scores of the LPF group, indicating a higher global health status and a higher quality of physical, emotional, and cognitive functioning in the LPF group. The other functional scales of the EORTC QLQ-C30 (role functioning and social functioning) and the functional scales of the EORTC QLQ-CR29 (body image, anxiety, and weight) showed a negative reconstruction effect. This shows a trend towards higher scores of the control group, indicating a higher quality of role and social functioning, higher body image, less anxiety, and less weight problems in the control group; however none of these differences were significant in our small study population.

\section{Sexual Functioning}

In the LPF group, 12 of the 15 patients had a partner (80\%) and 13 of the 15 patients were sexually active before surgery $(86.7 \%)$. Five patients $(33.3 \%)$, one female and four males, reported being sexually active postoperatively; all five were also sexually active preoperatively. Four (26.7\%) of these patients, one female and three males, had intercourse after surgery. Of the three patients who completed the FSFI/MSFI, the median total score was 16.8 (15.2-30.1). One patient scored above the cut-off point of 26 for normal sexual functioning.

In the control group, nine patients (56.3\%), seven males and two females, were sexually active postoperatively.
FIG. 1 A 55-year-old male patient. (a) Defect following ELAPE; (b) harvest of lotus petal flap; (c) direct postreconstruction; (d) 11 days post-reconstruction;

(e) 10 weeks post-

reconstruction; (f) 2 years postreconstruction. ELAPE extralevator abdominoperineal excision

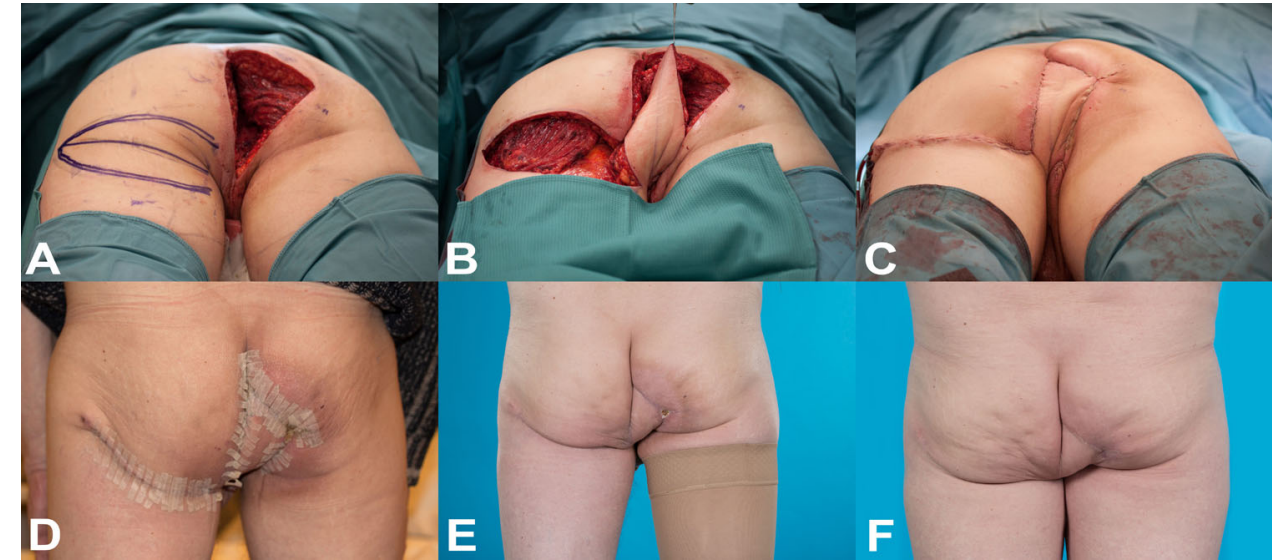


TABLE 2 EORTC QLQ-C30 and EORTC QLQ-CR29 scores

\begin{tabular}{|c|c|c|c|c|c|c|c|}
\hline & \multicolumn{2}{|c|}{ Lotus petal flap group $[n=15]$} & \multicolumn{2}{|c|}{ Control group $[n=16]$} & \multirow[t]{2}{*}{ Reconstruction effect $(\beta)$} & \multirow[t]{2}{*}{$95 \% \mathrm{CI}$} & \multirow[t]{2}{*}{$p$-Value } \\
\hline & Median & Range & Median & Range & & & \\
\hline \multicolumn{8}{|l|}{ EORTC QLQ-C3O } \\
\hline Global health status & 66.7 & $33.3-100$ & 79.2 & $33.3-100$ & 8.0 & -17.0 to 33.0 & 0.51 \\
\hline \multicolumn{8}{|l|}{ Functional scales } \\
\hline Physical functioning & 66.7 & $26.7-100$ & 80.0 & $0.0-100$ & 6.7 & -19.3 to 32.7 & 0.60 \\
\hline Role functioning & 50.0 & $16.7-100$ & 83.3 & $0.0-100$ & -11.5 & -43.8 to 20.7 & 0.47 \\
\hline Emotional functioning & 75.0 & $25.0-100$ & 95.8 & $0.0-100$ & 3.7 & -32.0 to 39.4 & 0.83 \\
\hline Cognitive functioning & 83.3 & $16.7-100$ & 83.3 & $16.7-100$ & 3.3 & -29.6 to 36.1 & 0.84 \\
\hline Social functioning & 66.7 & $16.7-100$ & 100 & $0.0-100$ & -6.8 & -44.1 to 30.5 & 0.71 \\
\hline \multicolumn{8}{|l|}{ EORTC QLQ-CR29 } \\
\hline \multicolumn{8}{|l|}{ Functional scales } \\
\hline Body image & 33.3 & $0.0-100$ & 77.8 & $0.0-100$ & -18.1 & -61.5 to 25.3 & 0.40 \\
\hline Anxiety & 66.7 & $0.0-100$ & 83.3 & $0.0-100$ & -18.3 & -63.0 to 26.4 & 0.41 \\
\hline Weight & 100 & $0.0-100$ & 100 & $0.0-100$ & -6.0 & -44.9 to 32.9 & 0.76 \\
\hline
\end{tabular}

A high score represents a high quality of life or a high level of functioning

CI confidence interval, EORTC QLQ-CR29 European Organization for Research and Treatment of Cancer Quality of Life Questionnaire CR29, EORTC QLQ-C30 European Organization for Research and Treatment of Cancer Quality of Life Questionnaire C30

\section{Lotus Petal Flaps-Specific Physical Functioning}

Six patients underwent a reconstruction with a unilateral $(54.5 \%) \mathrm{LPF}$, four of whom reported no limitations in hip function on either side. Two patients reported limitations on both sides during sitting. Five patients underwent a bilateral LPF reconstruction (45.5\%), one of whom reported no limitations, two reported limitations on both sides during sitting and activities, and two reported limitations on just one side during activities. All except one of the included patients experienced limitations in the duration of sitting, varying from 1 to $60 \mathrm{~min}$. Five patients were no longer able to ride a bicycle following reconstruction (three for a limited duration, varying from 2 to $60 \mathrm{~min}$ ), and three patients experienced no limitations during cycling. During physical testing, the ROM of the LPF group had a median maximum flexion of $105^{\circ}$ (range 80-130) and a median maximum (hyper)extension of $10^{\circ}$ (range 0-25). No signs of perineal herniation were found in any of the 11 patients who underwent reconstruction.

\section{DISCUSSION}

The aim of this study was to evaluate QoL, sexual functioning, and physical functioning following perineal reconstruction using the LPF technique. The main results of our study show that despite the extent of the resection performed in the LPF group, QoL was not significantly different from that of patients with a smaller resection, in whom there was no need for reconstruction using flaps. Earlier studies on perineal reconstruction following resection for rectal cancer compared the use of the vertical rectus abdominis myocutaneous (VRAM) flap with primary closure. Those studies showed no or only small differences between both groups on the EORTC QLQ-C30 and EORTC QLQ-CR29. ${ }^{23,24}$ QoL studies in the field of oncology often reveal little changes in QoL, and Sprangers and Schwartz explained this phenomenon as response shift, ${ }^{25}$ which means that a life-threatening disease, such as cancer, may change a patient's internal standards of selfevaluation. Therefore, the experience with cancer may change their expectations about life and, as such, they may evaluate their QoL according to these new expectations and internal standards, resulting in the report of a relatively good QoL despite the large impact of this major life event. $^{25-27}$

The results also indicated that, in retrospect, patients who underwent LPF reported decreased sexual and physical functioning. Moreover, only one patient scored above the cut-off of normal sexual functioning postoperatively. Low sexual functioning could be partially due to the reconstruction, but also the malignant disease itself may have influenced sexual functioning. Low sexual functioning has been reported in earlier studies on preoperative patients diagnosed with colorectal carcinoma, in which the mean and median scores of patients were well below the cut-off of normal sexual functioning. ${ }^{28,29}$ No earlier studies on sexual functioning following LPF reconstruction have 
been reported. An earlier study on postoperative sexual functioning after perineal resection comparing patients without reconstruction with patients who underwent reconstruction using the VRAM technique showed that the non-reconstruction group had slightly better sexual functioning (mean 21.0 vs. 12.9). ${ }^{30}$ It should be noted though that this study, like ours, was performed in a small group $(n=29)$, which limits the reliability of the findings. However, given the consistency in findings, it does stress the importance of discussing sexual functioning during preoperative counseling since the reconstruction seemed to have an impact on sexual functioning. Indeed, in their systematic review on sexual function following perineal reconstruction, McArdle et al. ${ }^{31}$ also emphasized the importance of preoperative counseling to ensure realistic goals and expectations on postoperative sexual functioning.

Regarding LPF-specific physical functioning, many patients reported limitations during sitting, especially those patients after bilateral use of LPF. The median ROM of the hip $\left(105^{\circ}\right)$ was lower compared with the average ROM of healthy people $\left(120^{\circ}\right)$, as published by the American Academy of Orthopaedic Surgeons; ${ }^{22}$ however, this degree of flexion is still considered normal and does not cause any impairment. ${ }^{32}$ In addition, the hip extension was decreased following LPF reconstruction $\left(10^{\circ}\right)$ compared with untreated people $\left(30^{\circ}\right) .{ }^{22}$ Hypothetically, the donor site on the posterior side of the hip may cause (temporary) tightness of the skin and limit flexion of the hip. In that case, only a decrease in flexion would have been expected. However, in our study group, limitation during both flexion and extension was seen. The decreased ROM (both flexion and extension) could possibly be due to either the radiotherapy or to pain.

During the outpatient clinic visits, no signs of perineal herniation were found in any of the 11 patients. The incidence of perineal herniation following ELAPE without use of a flap ranges from 1 to $26 \%$. Our outcome is comparable with the systematic review of Balla et al. ${ }^{12}$ on perineal hernia repair after perineal resection, who also reported low recurrence rates of perineal herniation when a flap was used. Use of an omentoplasty or biological mesh also contributes to the prevention of perineal herniation. Reconstruction using the LPF seems to have a limited influence on the ROM and to prevent perineal herniation.

\section{Limitations}

This study had a small study population and a broad range in time between surgery and survey. This is hard to overcome as the application of this reconstruction technique is limited and, in addition, many patients unfortunately have a limited life expectancy following treatment of advanced rectal and anal cancer. Cases in the
LPF group had larger defect sizes than those in the control group. This selection bias cannot be avoided. Patients with defects large enough to require reconstruction cannot be closed primarily, and it would be unethical to reconstruct patients when their wound can be closed primarily. However, this is the largest study to date on the subject of QoL and sexual and physical functioning following perineal reconstruction with the LPF. Prospective multicenter studies, with a standardized presurgical assessment on QoL, sexual functioning, and physical functioning, are needed to increase the quality and quantity of data.

\section{CONCLUSION}

LPF reconstruction after extensive perineal resection did not impair QoL when compared with perineal resection without reconstruction, even though the resection size was much larger in the LPF group. Both sexual and physical functioning become, to a certain extent, impaired following LPF reconstruction in the perineal area, although not hampering overall satisfaction, and no cases of perineal herniation were observed.

ACKNOWLEDGMENTS The authors acknowledge M. El Moumni, $\mathrm{MD}, \mathrm{PhD}$, for his contribution to the statistical analysis.

OPEN ACCESS This article is licensed under a Creative Commons Attribution 4.0 International License, which permits use, sharing, adaptation, distribution and reproduction in any medium or format, as long as you give appropriate credit to the original author(s) and the source, provide a link to the Creative Commons licence, and indicate if changes were made. The images or other third party material in this article are included in the article's Creative Commons licence, unless indicated otherwise in a credit line to the material. If material is not included in the article's Creative Commons licence and your intended use is not permitted by statutory regulation or exceeds the permitted use, you will need to obtain permission directly from the copyright holder. To view a copy of this licence, visit http://creativecommons. org/licenses/by/4.0/.

DISCLOSURES PW is a scientific advisory board member for Fidia Ltd, Milan, Italy. Joke Hellinga, Martin W. Stenekes, Moniek Janse, Joke Fleer, and Boudewijn van Etten have no conflicts of interest to declare and no sources of funding to report.

\section{REFERENCES}

1. Butler CE, Gündeslioglu AO, Rodriguez-Bigas MA. Outcomes of immediate vertical rectus abdominis myocutaneous flap reconstruction for irradiated abdominoperineal resection defects. $J$ Am Coll Surg. 2008;206:694-703.

2. Mughal M, Baker RJ, Muneer A, Mosahebi A. Reconstruction of perineal defects. Ann R Coll Surg Engl. 2013;95:539-544.

3. Devulapalli C, Tong Jia Wei A, DiBiagio JR, et al. Primary versus flap closure of perineal defects following oncologic resection: a systematic review and meta-analysis. Plast Reconstr Surg. 2016;137:1602-1613. 
4. Khoo AK, Skibber JM, Nabawi AS, et al. Indications for immediate tissue transfer for soft tissue reconstruction in visceral pelvic surgery. Surgery. 2001;130:463-469.

5. Radice E, Nelson H, Mercill S, Farouk R, Petty P, Gunderson L. Primary myocutaneous flap closure following resection of locally advanced pelvic malignancies. Br J Surg. 1999;86:349-354.

6. Hellinga J, Khoe PCKH, van Etten B, Hemmer PH, Havenga K, Stenekes MW, Eltahir Y. Fasciocutaneous lotus petal flap for perineal wound reconstruction after extralevator abdominoperineal excision: application for reconstruction of the pelvic floor and creation of a neovagina. Ann Surg Oncol. 2016;23:4073-4079.

7. Yii NW, Niranjan NS. Lotus petal flaps in vulvo-vaginal reconstruction. Br J Plast Surg. 1996;49:547-554.

8. Bodin F, Dissaux C, Seigle-Murandi F, Dragomir S, Rohr S, Bruant-Rodier C. Posterior perineal reconstructions with "suprafascial" lotus petal flaps. J Plast Reconstr Aesthet Surg. 2015;68:e7-e12.

9. Pantelides NM, Davies RJ, Fearnhead NS, Malata CM. The gluteal fold flap: a versatile option for perineal reconstruction following anorectal cancer resection. J Plast Reconstr Aesthet Surg. 2013;66:812-820.

10. Winterton RI, Lambe GF, Ekwobi C, Oudit D, Mowatt D, Murphy JV, Ross GL. Gluteal fold flaps for perineal reconstruction. $J$ Plast Reconstr Aesthet Surg. 2013;66:397-405.

11. Aerts L, Enzlin P, Vergote I, Verhaeghe J, Poppe W, Amant F. Sexual, psychological, and relational functioning in women after surgical treatment for vulvar malignancy: a literature review. $J$ Sex Med. 2012;9:361-371.

12. Balla A, Batista Rodríguez, Buonomo N, Martinez C, Hernández P, Bollo J, Targarona EM. Perineal hernia repair after abdominoperineal excision or extralevator abdominoperineal excision: a systematic review of the literature. Tech Coloproctol. 2017;21:329-336.

13. Butt HZ, Salem MK, Vijaynagar B, Chaudhri S, Singh B. Perineal reconstruction after extra-levator abdominoperineal excision (eLAPE): a systematic review. Int $J$ Colorectal Dis. 2013;28:1459-1468.

14. Janse M, Sprangers MA, Ranchor AV, Fleer J. Long-term effects on goal disturbance and adjustment on well-being in cancer patients. Qual Life Res. 2016;25:1017-1027.

15. Aaronson NK, Ahmedzai S, Bergman B, et al. The European Organization for Research and Treatment of Cancer QLQ-C30: a quality-of-life instrument for use in international clinical trials in oncology. J Natl Cancer Inst. 1993;85:365-376.

16. Whistance RN, Conroy T, Chie W, et al. Clinical and psychometric validation of the EORTC QLQ-CR29 questionnaire module to assess health-related quality of life in patients with colorectal cancer. Eur J Cancer. 2009;45:3017-3026.

17. Stiggelbout AM, Kunneman M, Baas-Thijssen MC, et al. The EORTC QLQ-CR29 quality of life questionnaire for colorectal cancer: validation of the Dutch version. Qual Life Res. 2016;25:1853-1858.

18. Kalmbach DA, Ciesla JA, Janata JW, Kingsberg SA. The validation of the Female Sexual Function Index, Male Sexual Function Index, and Profile of Female Sexual Function for use in healthy young adults. Arch Sex Behav. 2015;44:1651-1662.
19. Rosen R, Brown C, Heiman J, et al. The Female Sexual Function Index (FSFI): a multidimensional self-report instrument for the assessment of female sexual function. J Sex Marital Ther. 2000;26:191-208.

20. Ter Kuile MM, Brauer M, Laan E. De Female Sexual Function Index (FSFI) en de Female Sexual Distress Scale (FSDS): psychometrische eigenschappen in een Nederlandse populatie. Tijdschrift voor Seksuologie. 2009;33:207-222.

21. Wiegel M, Meston C, Rosen R. The Female Sexual Function Index (FSFI): Cross-Validation and Development of Clinical Cutoff Scores. J Sex Marital Ther. 2005;31:1-20.

22. American Academy of Orthopaedic Surgeons (1965). Joint motion: method of measuring and recording. 4th reprint. Edinburgh: E. \& S. Livingstone Ltd; 1969.

23. Holman FA, Martijnse IS, Traa MJ, Boll D, Nieuwenhuijzen GA, de Hingh $\mathrm{IH}$, et al. Dynamic article: vaginal and perineal reconstruction using rectus abdominis myocutaneous flap in surgery for locally advanced rectum carcinoma and locally recurrent rectum carcinoma. Dis Colon Rectum. 2013;56:175-185.

24. O'Dowd V, Burke JP, Condon E, et al. Vertical rectus abdominis myocutaneous flap and quality of life following abdominoperineal excision for rectal cancer: a multi-institutional study. Tech Coloproctol. 2014;18:901-906.

25. Sprangers MAG, Schwartz CE. Integrating response shift into health-related quality of life research: a theoretical model. Soc Sci Med. 1999;48:1507-1515.

26. Donohoe JE. To what extent can response shift theory explain the variation in prostate cancer patients' reactions to treatment sideeffects? A review. Qual Life Res. 2011;20:161-167.

27. Schwartz CE. Applications of response shift theory and methods to participation measurement: a brief history of a young field. Arch Phys Med Rehabil. 2010;91(9):S38-S43.

28. Segelman J, Martling A, Machado M, Holm T, Bergmark K, Flöter Rådestad A. Preoperative sexual function in women with rectal cancer. Eur J Surg Oncol. 2013;39:1079-1086.

29. Traa MJ, De Vries J, Roukema JA, Den Oudsten BL. The preoperative sexual functioning and quality of life in colorectal cancer: a study among patients and their partners. J Sex Med. 2012;9:3247-3254.

30. Corte H, Lefèvre JH, Dehnis N, Shields C, Chaouat M, Tiret E, et al. Female sexual function after abdominoperineal resection for squamous cell carcinoma of the anus and the specific influence of colpectomy and vertical rectus abdominis myocutaneous flap. Colorectal Dis. 2011;13:774-778.

31. McArdle A, Bischof DA, Davidge K, Swallow CJ, Winter DC. Vaginal reconstruction following radical surgery for colorectal malignancies: a systematic review of the literature. Ann Surg Oncol. 2012;19:3933-3942.

32. Rondinelli RD, Genovesc E, Katz RT, et al. Guides to the Evaluation of Permanent Impairment. 6th edition. Chicago, IL: American Medical Association; 2008.

Publisher's Note Springer Nature remains neutral with regard to jurisdictional claims in published maps and institutional affiliations. 